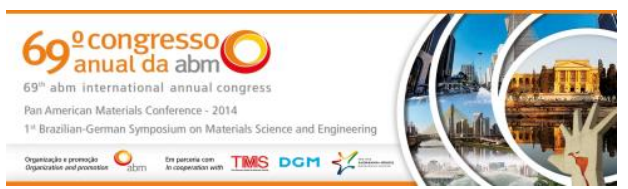

Tema: Iniciação científico-tecnológica

\title{
ANÁLISE DO COMPORTAMENTO DE FERRAMENTA DE METAL DURO NO FRESAMENTO COM ALTA VELOCIDADE DE CORTE NO AÇO AISI H13 ENDURECIDO*
}

\author{
Leonardo Roberto da Silva' \\ Manoel Amâncio Gomes² \\ Arthur Hermsdorff Cezar ${ }^{3}$ \\ Isabela Caroline Ribeiro da Silva ${ }^{3}$
}

\section{Resumo}

Com o desenvolvimento de novos materiais para ferramentas de corte, os processos de usinagem de aços endurecidos (com remoção de cavacos em aço com dureza superior a $45 \mathrm{HRC}$ ) têm estado cada vez mais em evidência, de tal modo que, em alguns casos, substitui-se o processo de retificação por possibilitar a redução do tempo de produção e do custo de fabricação. Além disso, é possível obter peças com elevado acabamento superficial e tolerâncias apertadas. Este trabalho visa analisar o desgaste de ferramenta de metal duro e possíveis danos causados na superfície da peça através das solicitações térmicas e mecânicas originadas do fresamento com alta velocidade de corte no aço AISI H13 endurecido. A avaliação do desempenho das ferramentas de metal duro consistiu na análise dos seguintes parâmetros: vida, desgaste de ferramenta, dureza, rugosidade e microestrutura. Os resultados obtidos neste estudo mostraram que o material da ferramenta e a velocidade de corte influenciaram no desempenho das variáveis de saída citadas. As ferramentas de metal duro apresentaram baixo rendimento na usinagem HSM (High Speed Machining) e rendimento satisfatório com velocidades de corte convencionais.

Palavras-chave: Usinagem com alta velocidade; Aços endurecidos; Rugosidade; Microestrutura; Desgaste de ferramenta.

\section{CARBIDETOOLANALYSISBEHAVIORIN HIGH SPEED MILLING OFHARDENED AISI H13 STEEL}

\begin{abstract}
Because of the development of new materials for cutting tools, machining processes of hardened steels (chip removal on steels with hardness above $45 \mathrm{HRC}$ ) have been increasingly in evidence, in a way that, in some cases replace the grinding process by allowing the reduction of production time and manufacturing cost. Furthermore, it is possible to obtain parts with high surface finish and tight tolerances. This article aims to analyze the wear of carbide tools and possible damages in the part surface through mechanical and thermal stresses in high speed milling of hardened steel AISI H13. Performance evaluation of carbide tools will be the analysis of the following parameters: life, tool wear, hardness, roughness and microstructure. Results in this article showed that the tool material and the cutting speed influenced the performance of the cited output variables. The carbide tools showed low performance in HSM machining and satisfactory performance in conventional cutting speeds.

Keywords: High speed machining; Hardened steels; roughness; microstructure; Tool wear

1 Professor Doutor, Engenharia Mecânica, Centro Federal de Educação Tecnológica de Minas Gerais (CEFET-MG), Belo Horizonte, MG, Brasil.

2 Mestrando, Programa de Pós-Graduação em Engenharia de Materiais, CEFET-MG, Belo Horizonte, MG, Brasil.

3 Graduando, Engenharia de Materiais, CEFET-MG, Belo Horizonte, MG, Brasil.
\end{abstract}

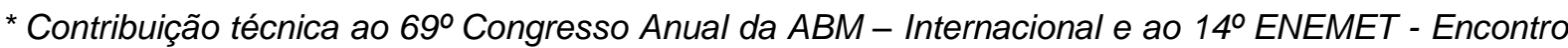
Nacional de Estudantes de Engenharia Metalúrgica, de Materiais e de Minas,21 a 25 de julho de 2014, São Paulo, SP, Brasil. 


\section{INTRODUÇÃO}

A usinagem de materiais duros vem sendo cada vez mais utilizada ao longo dos tempos, devido ao crescente aumento da demanda de produção e à necessidade de redução dos custos e prazos de fabricação, oriundos da exigência cada vez maior das novas tecnologias empregadas nos processos industriais. Convencionou-se chamar de usinagem de materiais duros, a remoção de cavacos em materiais com dureza superior a $45 \mathrm{HRC}$. Há poucos anos, materiais que necessitavam ser usinados em sua forma endurecida eram usinados apenas pelo processo de retificação. Hoje, devido ao desenvolvimento de materiais para ferramentas de elevada dureza e resistência ao desgaste em altas temperaturas, aliados ao surgimento de máquinas e ferramentas de maiores rigidez e precisão dimensional em altas rotações, já é possível a usinagem desses materiais pelos processos de fresamento e torneamento [1-4].

Segundo Gamarra [5], o fresamento é também a área clássica de aplicação para HSM (High Speed Machining). Tal processo permite o uso de velocidades de cinco a dez vezes maiores que as utilizadas na usinagem convencional [6-7]. Além da redução do tempo de produção ao se atingir tais velocidades, destacam-se ainda outras qualidades para HSM, como: altas taxas de remoção de material, baixas forças de corte, mínimas distorções nas peças, capacidade de usinar paredes finas, utilização de simples dispositivos de fixação, ótimos acabamentos superficiais, pequeno ou nenhum dano térmico à integridade da superfície da peça, peças livres de rebarbas e fácil manuseio dos cavacos [8].

Apesar das vantagens citadas, alguns problemas são encontrados no uso de HSM, como, por exemplo, a alta taxa de desgaste nas ferramentas e redução de vida útil das mesmas. Isso exige que algumas medidas sejam tomadas para que a razão custo-benefício ainda seja favorável às perspectivas de uma determinada indústria. Então, o material da ferramenta de corte deve ser duro e sua resistência ao calor elevada para que a vida útil esteja em um nível aceitável $[6,8]$.

O metal duro é considerado o material com maior aplicação na área de usinagem. Até algumas décadas atrás, vários autores consideravam que o mesmo apresentava uma série de limitações para aplicação em altas velocidades [9-11]. No entanto, a partir da década de 90, a indústria de moldes e matrizes passou a utilizá-lo com grande sucesso. Alguns dos motivos citados para que isso fosse observado foram a fabricação de ferramentas utilizando grãos menores que $1 \mu \mathrm{m}$ e a utilização de várias camadas de revestimentos [2]. Somado a isso, observou-se que as temperaturas alcançadas com HSM não eram tão elevadas quanto se imaginava, cerca de $200^{\circ}$ a $400^{\circ} \mathrm{C}$ contra os $1200^{\circ} \mathrm{C}$ da usinagem convencional [12]. Hoje ainda, vários pesquisadores [13-15] vêm observando um bom desempenho do metal duro em aplicações com aços endurecidos, principalmente em aço AISI H13.

Tönshoff et al. [16] afirmam que o desgaste da ferramenta e a integridade superficial das peças usinadas são fortemente influenciados pela utilização ou não de fluidos de corte, uma vez que eles agem no sentido de reduzir a carga térmica na aresta de corte, aumentando, assim, a vida da ferramenta quando comparado com o corte a seco. No entanto, observa-se que nos sistemas de lubri-refrigeração convencionais são utilizados milhares de litros de emulsões lubrificantes, que muitas vezes são descartados diretamente na natureza, levando a contaminação do solo e da água e gerando forte impacto ambiental. Além disso, o contato do operador das máquinas com o fluido pode gerar graves problemas de saúde. Para atender as exigências dos órgãos de controle ambiental, a maior parte das empresas que ainda utilizam fluidos

\footnotetext{
* Contribuição técnica ao $69^{\circ}$ Congresso Anual da ABM - Internacional e ao 14ํㅡㄹ ENEMET - Encontro Nacional de Estudantes de Engenharia Metalúrgica, de Materiais e de Minas,21 a 25 de julho de 2014, São Paulo, SP, Brasil.
} 


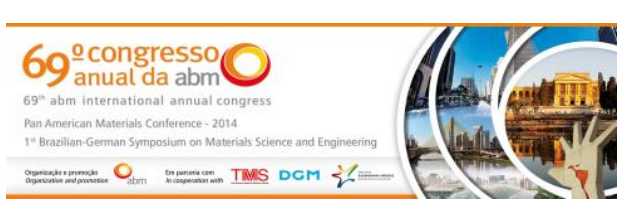

de corte tem investido no reprocessamento desses, o que gera um grande custo adicional, tornando cada vez mais cara sua utilização.

Dessa forma, a usinagem sem fluido de corte surge como uma tendência mundial, devido ao menor impacto ambiental, risco à saúde do operador e custo de produção. Essa ainda pode ser utilizada na tecnologia HSM, uma vez que o tempo de contato entre ferramenta e peça é pequeno, e consequentemente o calor gerado pelo atrito entre essas também, não se observando assim um aquecimento considerável [17].

Considerando todo o exposto, estão entre os objetivos do presente trabalho avaliar o desempenho da tecnologia de usinagem a seco no fresamento com alta velocidade de corte no aço AISI H13 endurecido em operação de acabamento, assim como o estudo do desgaste de ferramentas de metal duro e possíveis danos causados na superfície da peça através das solicitações térmicas e mecânicas originadas do processo sob tais condições de corte.

\section{MATERIAIS E MÉTODOS}

Os corpos de prova de dimensões $150 \times 18 \times 40 \mathrm{~mm}$ utilizados nos ensaios de usinagem foram produzidos, inicialmente, pelo processo de laminação, com dureza máxima de $207 \mathrm{HB}$, e então endurecidos pelos processos de têmpera e revenimento com dureza final de 46 HRC. A composição química e as propriedades físicas do material foram fornecidas pelo fabricante, mediante certificado de qualidade da análise química, conforme mostrado na Tabela 1.

Tabela 1. Composição Química em \% e Propriedades Físicas. Fonte: VILLARES METAIS, 2006.

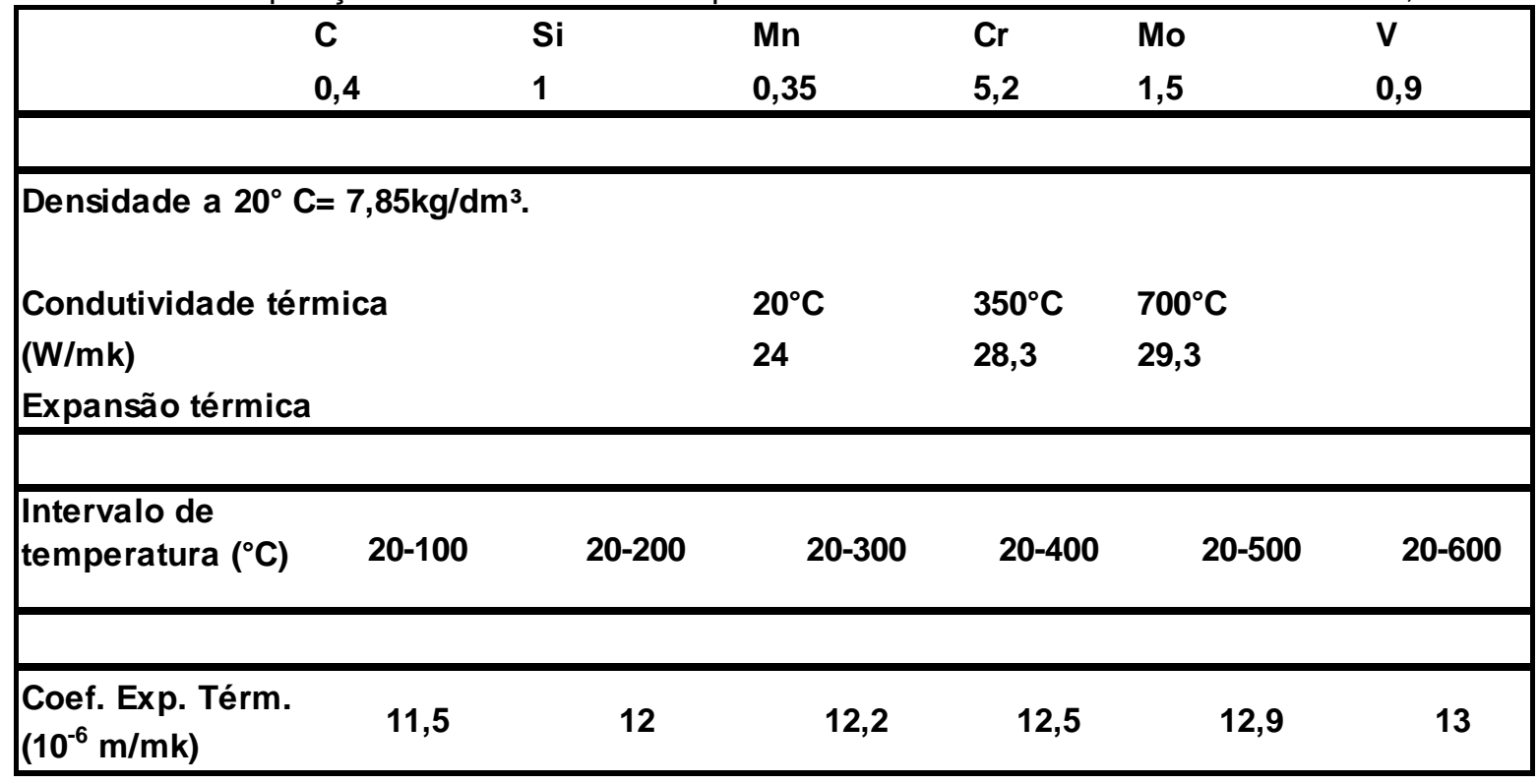

Os ensaios de usinagem foram realizados em uma fresadora MIKRON HSM 700 de $\mathrm{RPM}=\mathrm{MIN}$. $100 \mathrm{rpm}$ e MAX. $42.000 \mathrm{rpm}$ (Figura 1). Áreas de trabalho de $X=700 \mathrm{~mm} ; Y=550 \mathrm{~mm} ; Z=450 \mathrm{~mm}$ e capacidade de carga de mesa de até $700 \mathrm{~kg}$.

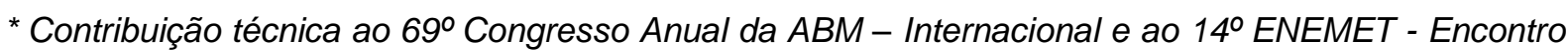
Nacional de Estudantes de Engenharia Metalúrgica, de Materiais e de Minas,21 a 25 de julho de 2014, São Paulo, SP, Brasil.
} 

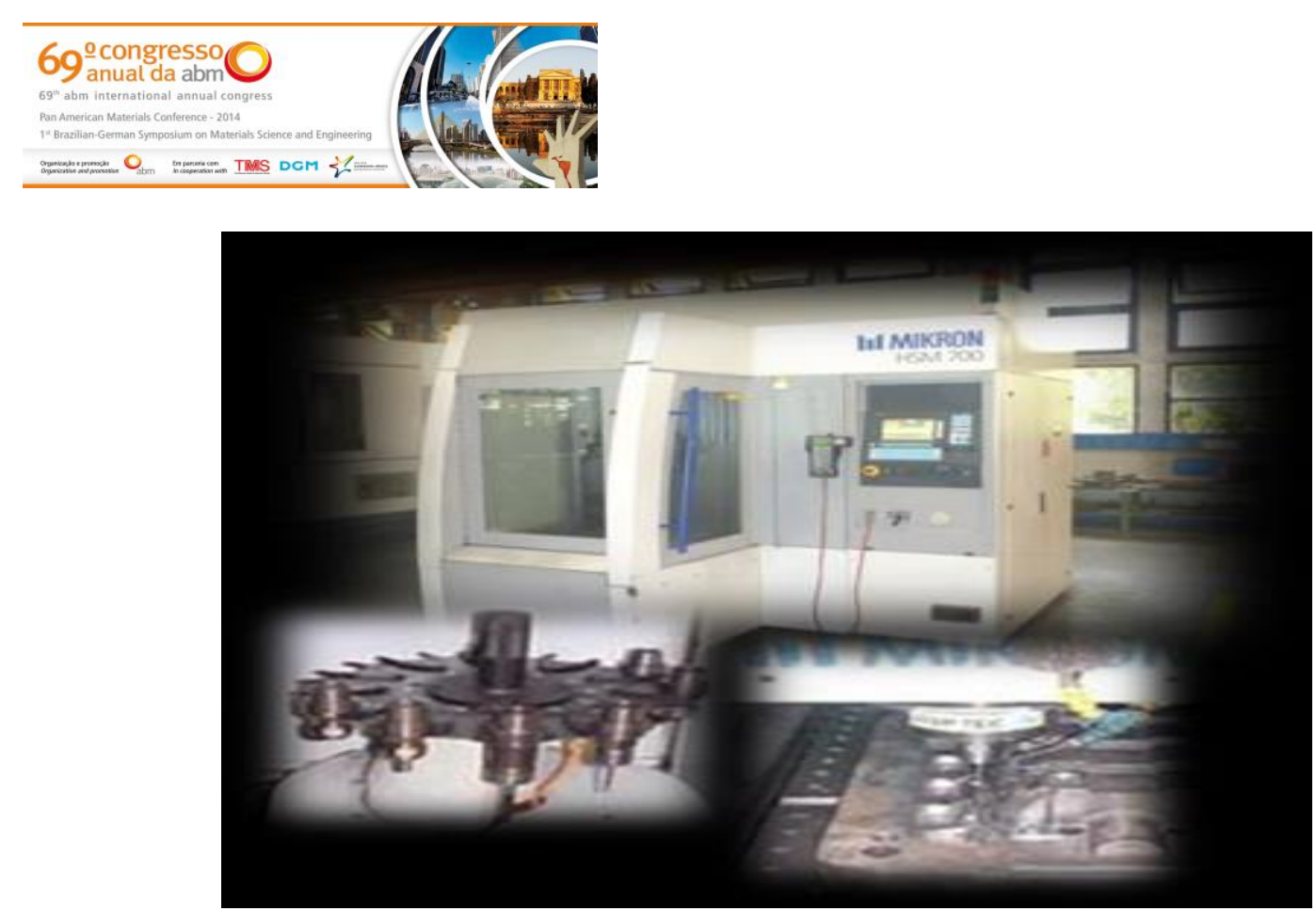

Figura 1. MIKRON HSM 700 CNC. Fonte: NEMAK Brasil, 2013.

Os corpos de prova foram fixados em uma morsa e usinados pela operação de fresamento frontal de acabamento, com uma fresa porta ferramenta de diâmetro $20 \mathrm{~mm}$ e com capacidade de montagem para duas ferramentas de corte.

A usinagem ocorreu em toda a área superficial da medida de $18 \mathrm{~mm}$, realizando os cortes discordante e concordante ao mesmo tempo.

Foram utilizadas ferramentas de metal duro classe F30M, cuja composição contém elementos de liga como W (Tungstênio), Co (Cobalto), Cr (Cromo) e C (Carbono) e uma cobertura composta de Ti (Titânio), Al (Alumínio) e N (Nitrogênio) de código ISO XOMX 120408TR-D14 (Figura 2).

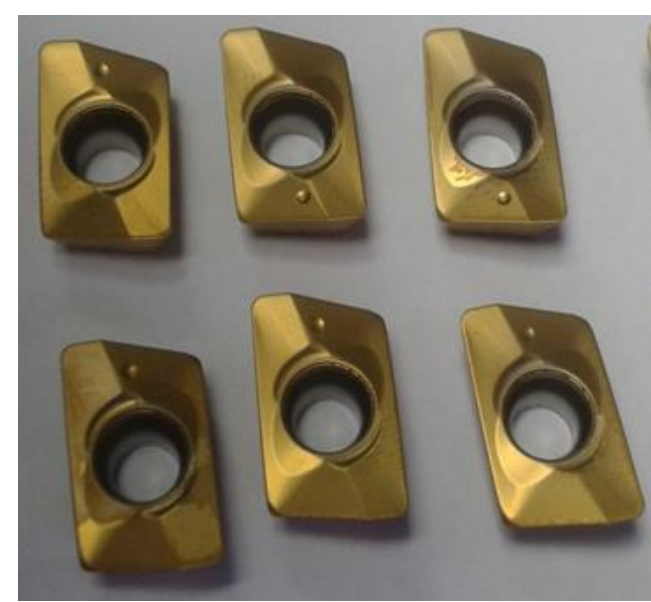

Figura 2. Imagem do inserto utilizado.

Os ensaios foram realizados com três velocidades de corte diferentes $(150 \mathrm{~m} / \mathrm{min}$, $225 \mathrm{~m} / \mathrm{min}$ e $300 \mathrm{~m} / \mathrm{min}$ ), mantendo-se $a_{p}$ (profundidade de corte) e $f_{z}$ (avanço por aresta) constantes e iguais a 0,2 $\mathrm{mm}$ em ambos os casos.

Para o primeiro valor de velocidade de corte utilizado, foram também usados os seguintes parâmetros: velocidade de avanço igual a $955 \mathrm{~mm} / \mathrm{min}$ e número de rotações por minuto igual a 2.387 .

Para o segundo valor de velocidade de corte utilizado, os parâmetros foram: velocidade de avanço igual a $1.432,5 \mathrm{~mm} / \mathrm{min}$ e número de rotações por minuto igual a 3.580 .

\footnotetext{
* Contribuição técnica ao $69^{\circ}$ Congresso Anual da ABM - Internacional e ao 14ํㅡㄹ ENEMET - Encontro Nacional de Estudantes de Engenharia Metalúrgica, de Materiais e de Minas,21 a 25 de julho de 2014, São Paulo, SP, Brasil.
} 

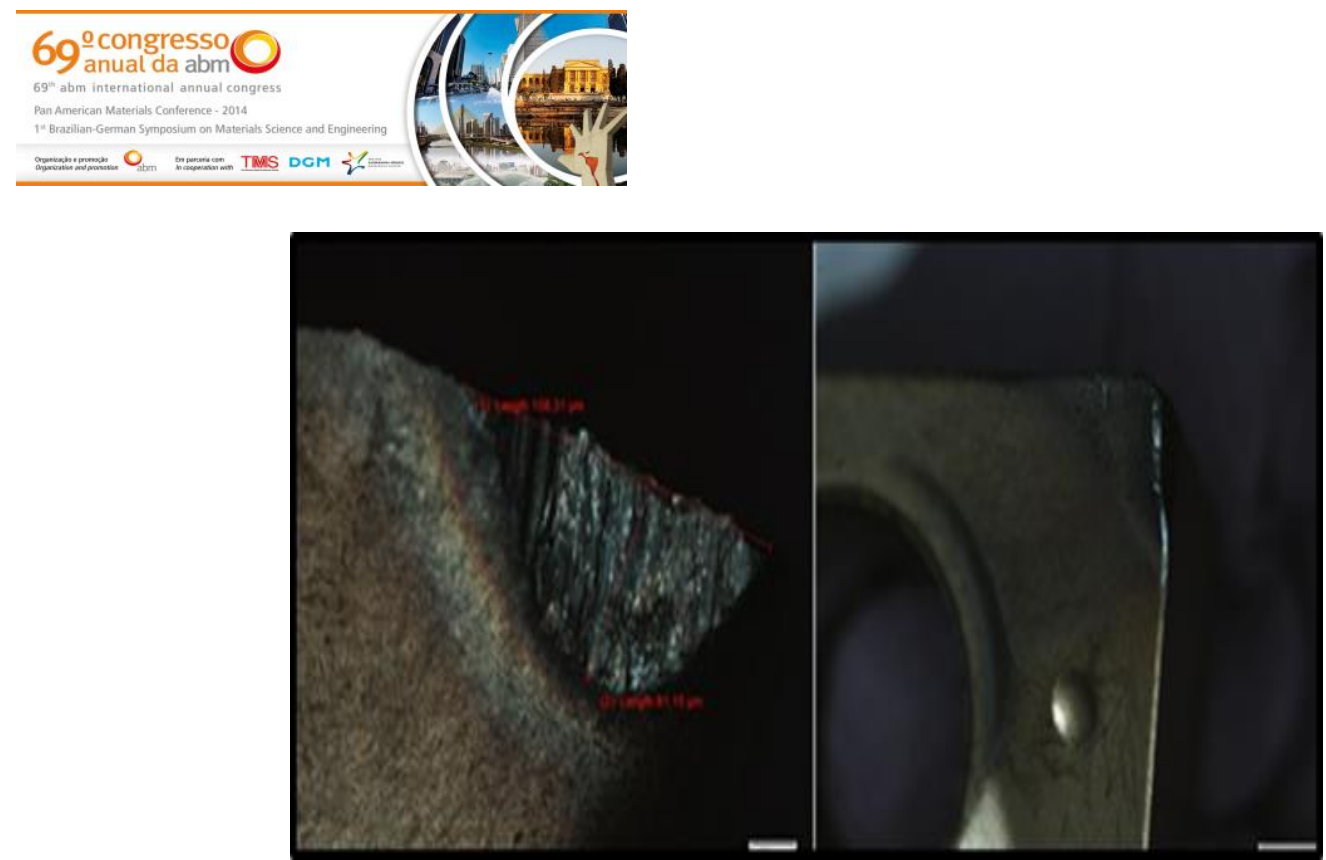

Figura 3. Desgaste observado na ferramenta para $\mathrm{Vc}_{\mathrm{c}}=225 \mathrm{~m} / \mathrm{min}$ (Ampliação de 50x).

De uma maneira geral, os desgastes que ocorreram na ferramenta de metal duro não são provenientes de um único mecanismo, mas sim de uma combinação de vários deles.

\subsection{Dureza}

Em todos os testes observados, não foram constatadas mudanças significativas na dureza dos corpos de prova, mantendo-se então o valor de 46 HRC durante a usinagem.

\subsection{Rugosidade}

A Tabela 3 apresenta os valores do parâmetro Ra encontrados para os três casos analisados após o último passe de usinagem.

Tabela 3. Rugosidade média (Ra) encontrada para diferentes velocidades de corte.

$$
\begin{aligned}
& \begin{array}{l}
\text { Velocidade de } \\
\text { corte }(\mathrm{m} / \mathrm{min})
\end{array} \\
& \quad \mathrm{Ra}(\mu \mathrm{m})
\end{aligned}
$$

\begin{tabular}{ll}
\hline 150 & 0,662 \\
\hline 225 & 0,557 \\
\hline 300 & 1,103 \\
\hline
\end{tabular}

É possível observar que o acabamento superficial apresentou valores de $\mathrm{Ra}$ menores com velocidades intermediárias.

\subsection{Microestrutura}

Pôde-se observar que o material passou por um processo de têmpera e revenimento, o que resultou em uma microestrutura martensítica, na qual foi possível observar os contornos de grãos do material.

A remoção do material durante a usinagem não causou alterações visíveis na microestrutura do material.

\footnotetext{
* Contribuição técnica ao 69ำ Congresso Anual da ABM - Internacional e ao 14ํㅡㄹ ENEMET - Encontro Nacional de Estudantes de Engenharia Metalúrgica, de Materiais e de Minas,21 a 25 de julho de 2014, São Paulo, SP, Brasil.
} 

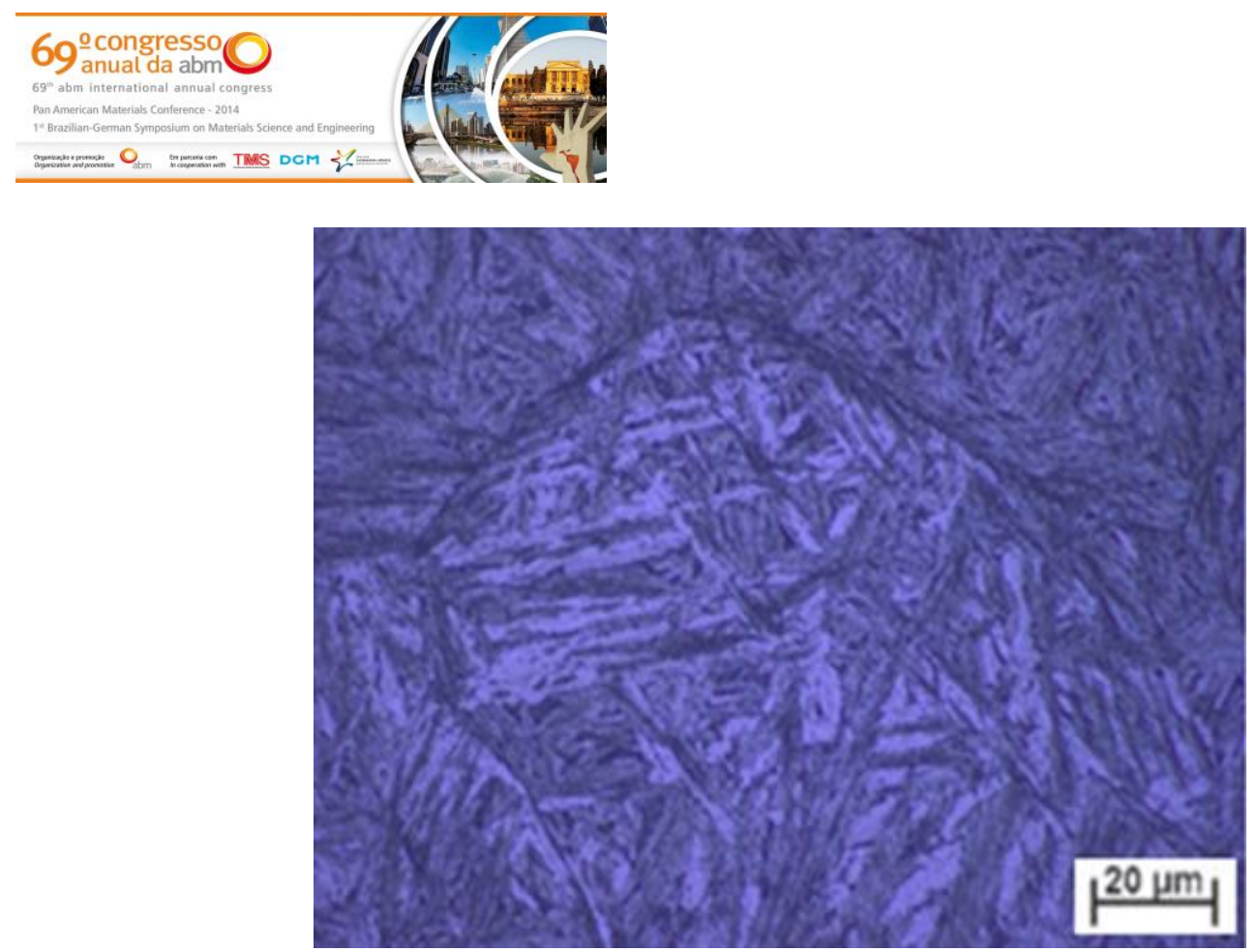

Figura 5. Microestrutura do aço H13 após tempera e revenimento (Ampliação de 500x).

\section{CONCLUSÃO}

A avaliação do desgaste da ferramenta de metal duro, da rugosidade, da dureza e da microestrutura dos corpos de prova levou às seguintes conclusões:

- Na maior parte dos processos de usinagem, é observado mais de um tipo de desgaste;

- Não é possível definir com precisão o tipo de desgaste predominante;

- A velocidade de corte tem influência direta na vida da ferramenta, na rugosidade e no desgaste da ferramenta;

- A dureza tem influência direta na usinabilidade do material.

\section{Agradecimentos}

Ao CNPq pela bolsa de Produtividade em Pesquisa e pelo financiamento das bolsas de Iniciação Científica.

Demais agradecimentos à empresa Nemak por permitir a realização dos ensaios, ao engenheiro Ricardo que colaborou na análise das amostras, e ao supervisor Nilson que auxiliou na usinagem das mesmas.

\section{REFERÊNCIAS}

1 Abrão AM, Aspinwall DK, Wise ML. A review of policrystalline cubic boron nitride tool developments and aplication. In: $30^{\text {th }}$ International MATADOR Conference. Manchester : UMIST; 1993. 1-11.

2 Dewes RC, Aspinwall DK. A review of ultra high speed milling of hardened steels. Journal of Materials Processing Technology, 1997; 69: 1-17.

3 Momper,FJ. Usinagem a seco e de materiais endurecidos. Máquinas e Metais, 2000; 411: 30-37.

4 Grzesik W. Influence of tool wear on surface roughness in hard turning using differently shaped ceramic tools. Wear. 2008; 265: 327-335.

5 Gamarra JR. Características das Ferramentas em HSM - Fresamento, Furação e Faceamento. In: Santos, A.V.et al. Usinagem em altíssimas velocidades: como os

\footnotetext{
* Contribuição técnica ao $69^{\circ}$ Congresso Anual da ABM - Internacional e ao 14ํㅡㄹ ENEMET - Encontro Nacional de Estudantes de Engenharia Metalúrgica, de Materiais e de Minas,21 a 25 de julho de 2014, São Paulo, SP, Brasil.
} 
